\title{
Evaluation of Language in Post-CVA Patients: A Clinicophonological Study
}

\author{
Anton Dev X ${ }^{1}$, Prithvi Das ${ }^{2}$, Somnath Saha ${ }^{3}$
}

\begin{abstract}
Aims and objectives:

- To assess patients with cerebrovascular accidents (CVA), in order to ascertain the presence and types of language deficits.

- Assess for any possible association between lesion site and pattern and degree of language impairments.

- To determine if the degree of language deficits are more in left hemisphere pathology.

Materials and methods: An observational study was conducted over a period of 1 year in the Department of ENT and Head-Neck Surgery and Department of Neuromedicine in a Tertiary Care Hospital. Poststroke patients were evaluated. Stable patients were selected. Both hemorrhagic and ischemic stroke patients were included. We had used the Western Aphasia Battery (WAB) to assess the faculty of Language in the study population and computed the Aphasia Quotient, based on which we categorized language function.

Results: In our study, the number of patients with Infarction as the pathologic process was greater than those with hemorrhage. A total of $40 \%$ of cases had exclusively subcortical lesions. In our study, young stroke (stroke in patient under 50 years of age), was present in $14 \%$. Aphasia was present in $40 \%$ of the patients. Crossed aphasia was seen in one patient (4\%). Based on the aphasia quotient obtained from WAB, we categorized the aphasia as mild (4\%), moderate (12\%), and severe (24\%). We had a preponderance of nonfluent aphasia.

Conclusion: The frequency of poststroke aphasia was higher in our study (40\%) than that seen in earlier studies. We have seen a preponderance of nonfluent aphasia in our study with global aphasia $(50 \%)$ being the commonest subtype. Significant association $(p<0.05)$ was found between left-sided lesions and the presence of aphasia.

Keywords: Aphasia, Post-CVA, Poststroke, WAB.

International Journal of Phonosurgery \& Laryngology (2021): 10.5005/jp-journals-10023-1229
\end{abstract}

\section{INTRODUCTION}

In the US, stroke is the most significant contributor to long-term adult disability and the 5th most common cause of death.,

Disability is multifactorial and is determined by the site of the lesion, the degree of neurological recovery, premorbid status, and the environmental support systems.

\section{Localization of the Stroke}

In certain stroke varieties, there is a proclivity to occur in particular areas. For example: Lacunar stroke in the Subcortex. ${ }^{3}$ Damage occurring in one brain region impairs the specialized centers in the affected region and also loss of input from the injured part affects the entire brain. Arterial territories are used to localize stroke.

\section{Arterial Territory-errors Mapping}

- Contralateral hemiparesis/hemiplegia

- Contralateral sensory loss: ACA

- Contralateral homonymous hemianopia

- Left hemispheric: aaphasia

- Right hemispheric:Visual perceptual deficits including left neglect

- Contralateral hemiparesis/hemiplegia

- Contralateral sensory loss: MCA(Superior Division)

- Left hemispheric: Expressive aphasia

- Right hemispheric: Visual perceptual disorders

- Superior quadrantanopia or homonymous hemianopia MCA (Inferior division):

- Left hemispheric: Wernicke's aphasia

- Right hemispheric: Left visual neglect.
${ }^{1-3}$ Department of ENT, Calcutta National Medical College and Hospital, Kolkata, West Bengal, India

Corresponding Author: Prithvi Das, Department of ENT, Calcutta National Medical College and Hospital, Kolkata, West Bengal, India, Phone: +91 7439211782, e-mail: prithvidas89@gmail.com

How to cite this article: X Anton Dev, Das P, Saha S. Evaluation of Language in Post-CVA Patients: A Clinicophonological Study. Int J Phonosurg Laryngol 2021;11(2):54-60.

Source of support: Nil

Conflict of interest: None

Right Hemisphere Disorders

Injury to the right hemisphere results in:

- Visual-spatial Perceptual Disorders

Left-sided hemi-neglect, constructional apraxia, and asterognosis.

- Emotional Disorders

Indifference or flat affect, impulsivity, and emotional lability. Lack of insight is characteristic.

\section{- Communication Problems}

Aphasia is common in left hemispheric strokes, however, Aphasia has been observed after right hemispheric strokes in $30 \%$ of left-handed people and $5 \%$ of right-handed people. ${ }^{4}$

\section{Left Hemisphere Disorders}

Injury to left hemisphere results in:

- Aphasia

- Apraxias

() The Author(s). 2021 Open Access This article is distributed under the terms of the Creative Commons Attribution 4.0 International License (https://creativecommons. org/licenses/by-nc/4.0/), which permits unrestricted use, distribution, and non-commercial reproduction in any medium, provided you give appropriate credit to the original author(s) and the source, provide a link to the Creative Commons license, and indicate if changes were made. The Creative Commons Public Domain Dedication waiver (http://creativecommons.org/publicdomain/zero/1.0/) applies to the data made available in this article, unless otherwise stated. 
- Emotional disorders: Depression is seen after stroke in $50 \%$ of patients ${ }^{5}$ and is more common with frontal damage.

\section{Poststroke Aphasia}

Of all the impairments in stroke, aphasia is both common and highly debilitating. ${ }^{6,7}$ Aphasia has a prevalence of about $21-38 \%$, following acute stroke. A majority of individuals are right-handed (93\%), of whom the left hemisphere is dominant for language in $99 \%$. Language control in left-handed individuals is in the left hemisphere in $70 \%$, in the right hemisphere in $15 \%$, and in both hemispheres in $15 \% .{ }^{8}$ Hence, $97 \%$ of the population has the seat of language control in the left hemisphere (Table 1).

\section{Assessment of Aphasia Poststroke}

Diagnostic criteria for aphasia vary among different studies ${ }^{9}$ resulting in variations in statistics. Stroke studies show that language pathology is seen in $15^{10}-42 \%{ }^{11,12}$ patients of acute stroke. The number of first-time stroke patients recorded in 2013, was about 10.3 million. ${ }^{13}$ With such a high global burden, the accurate validation of poststroke aphasia assessments is vital for resource allocation. ${ }^{9}$

Neuroimaging reveals a correlation between lesion site and aphasia occurrence with recovery being dependably predicted based on lesion site and size. ${ }^{14,15}$ Imaging methods only characterize the lesion ${ }^{16}$ and further defining of language impairment will require additional bedside tests and clinical evaluation. ${ }^{17}$

Commonly used stroke scales are:

- The ESS- European Stroke Scale ${ }^{18}$

- CNS-Canadian Neurological Scale $e^{19}$

- NIHSS- National Institutes of Health Stroke Scale. ${ }^{20}$

Many of these scales have not been specifically validated for use in hyperacute stroke, despite being used for managing the same. ${ }^{21}$
Brief screening tools such as the Frenchay screening test, ${ }^{22}$ may be used by health professionals who are not specialists, ${ }^{21,24,25}$ to identify and promptly refer patients who are at-risk. ${ }^{21,22-25}$ Such tools usually do not test writing and reading. ${ }^{23}$ They are thereby not comprehensive and thereby not useful in isolation, for diagnosis. ${ }^{26}$ Other common, more elaborate speech pathology tools are:

- Acute aphasia screening protocol ${ }^{27}$

- Western aphasia battery-bedside ${ }^{28}$

- Mount wilga high-level language test ${ }^{29}$

- Sklar aphasia scale ${ }^{30}$

- Aachen aphasia bedside test ${ }^{31}$

- The Bedside evaluation screening test (BEST-2) $)^{32}$

- Aphasia language performance scales ${ }^{33}$

- Aphasia screening test. ${ }^{34}$

The shortfall of these tests is that their metrics are reported in their test manuals and not within peer-reviewed journals.

WAB has been utilized in our study.

\section{Western Aphasia Battery (WAB)}

The WAB is a Language assessment tool, used to test for Aphasia in patients between 18 and 89 years of age. The WAB has positive attributes such as validity and high consistency. ${ }^{35}$ The WAB, by design, has several subtest criteria and tests all language modalities. It also tests "higher cortical functioning." Pearson Assessments published the revised edition (WAB-R) in 2006, which also included a bedside screening test (Table 2).

\section{Materials and Methods}

\section{Study Design}

An observational study was done at a tertiary center in West Bengal.

\section{Study Population}

Patients admitted to the Neurology ward.

Table 1: Characteristic features of types of aphasia

\begin{tabular}{lccc}
\hline Type & Fluency & Comprehension & Repetition \\
\hline Broca's & Nonfluent & Good & Poor \\
Transcortical motor & Nonfluent & Good & Good \\
Wernicke's & Fluent & Poor & Poor \\
Transcortical sensory & Fluent & Poor & Good \\
Global & Nonfluent & Poor & Poor \\
Conduction & Fluent & Good & Poor \\
\hline
\end{tabular}

Table 2: Taxonomy of WAB

\begin{tabular}{lcccc}
\hline & \multicolumn{3}{c}{ Criteria for classification } \\
\hline Global & Fluency & Comprehension & Repetition & Naming \\
Broca's & $0-4$ & $0-3.9$ & $0-4.9$ & $0-6$ \\
Isolation & $0-4$ & $4-10$ & $0-7.9$ & $0-8$ \\
Transcortical motor & $0-4$ & $0-3.9$ & $5-10$ & $0-6$ \\
Wernicke's & $0-4$ & $4-10$ & $8-10$ & $0-8$ \\
Transcortical sensory & $5-10$ & $0-6.9$ & $0-7.9$ & $0-9$ \\
Conduction & $5-10$ & $0-6.9$ & $8-10$ & $0-9$ \\
Anomic & $5-10$ & $7-10$ & $0-6.9$ & $0-9$ \\
\hline
\end{tabular}




\section{Sample Design}

\section{Inclusion criteria}

Cerebrovascular accident (CVA) patients, including:

- Patients with ischemic stroke.

- Patients with hemorrhagic stroke.

- Medically stable patients.

\section{Exclusion Criteria}

- Recurrent stroke.

- Hemodynamically unstable patients.

- Unconscious patients.

- Subarachnoid hemorrhage.

- Agitation and possible inability to cooperate.

- Severe movement disorders (Dyskinesia)

- Patients with any other progressive neurological condition such as parkinsonism, schizophrenia.

- Mentally impaired patients who cannot provide proper informed consent and who would be uncooperative for examination.

- Patients of childhood stroke syndromes with various underlying genetic defects are excluded as they may skew the inferences, as a result of the differences in their baseline physiology.

\section{Sample Size}

- Total 50 cases.

\section{Study Tools}

- History including Past History of any Cerebrovascular event.

- Basic Hematology includes Complete blood count, platelets, $\mathrm{BT} / \mathrm{CT}$, urea, creatinine, and serology.

- Evaluation of Mode of communication (verbal/nonverbal).

- Western Aphasia Battery (WAB) for language status evaluation.

\section{Study Technique}

- This is a time-bound cross-sectional study.
- The study was done for a period of 1 year.

- Minimum of 50 post CVA patients will be selected consecutively based on inclusion and exclusion criteria.

- The region of CNS involved will be ascertained based on the expert radiological opinion of the imaging that would have already been done and if documentation is unavailable, the necessary radiological imaging will be performed.

- Language function will be assessed clinically, to look for the presence and type of defect if any, and then using the WAB scoring system the degree of impairment of language will be ascertained in the four dimensions of fluency, auditory-verbal comprehension, repetition, and naming. Subscores will be based on the taxonomic table. Accordingly, aphasia can be classified and an Aphasia quotient (AQ) can be determined. The language was classified as normal if an $A Q$ of 93.8 or above was achieved.

The findings of our study have been summarized in (Tables 3 to 9). (Fig. 1) represents the ages of subjects in the present study. (Fig. 2) represents the relative percentages of the various types of Aphasia we had observed in the present study. (Fig. 3) depicts the percentage of CVA patients who had normal speech and those with varying degrees of Aphasia as determined by Aphasia Quotient. (Fig. 4) represents the significant association observed between Left sided lesions and presence of Aphasia.

Table- 4 states the relationship with type of pathologic process with presence of Aphasia. There was no statistically significant relationship present $(p>0.05)$

From Table-5, we infer that the parameters were not associated with each other $(p>0.05)$

Table 3: Frequency of different cerebrovascular insult in our study

\begin{tabular}{lcc}
\hline Insult classification & Frequency & $\%$ \\
\hline Hemorrhage & 16 & 32.0 \\
Infarct & 34 & 68.0 \\
Total & 50 & 100.0 \\
\hline
\end{tabular}

Table 4: Type of pathologic process_-presence of aphasia

\begin{tabular}{lccccccc}
\hline & \multicolumn{2}{c}{ Present } & \multicolumn{2}{c}{ Absent } & \multicolumn{2}{c}{ Total } & \\
\cline { 2 - 8 } Insult & No & $\%$ & No & $\%$ & No & $\%$ & Results \\
\hline Hemorrhage & 6 & 12.0 & 10 & 20.0 & 16 & 32.0 & $\mathrm{X}^{2}=0.061$ \\
Infarct & 14 & 28.0 & 20 & 40.0 & 34 & 68.0 & $\mathrm{df}=1$ \\
Total & 20 & 40.0 & 30 & 60.0 & 50 & 100.0 & $p=0.804$ \\
\hline
\end{tabular}

Table 5: Site of lesion with type of aphasia

\begin{tabular}{|c|c|c|c|c|c|c|c|c|c|}
\hline \multirow[b]{2}{*}{ Type } & \multicolumn{2}{|c|}{ Cortical } & \multicolumn{2}{|c|}{ Corti-cosubcortical } & \multicolumn{2}{|c|}{ Subcortical } & \multicolumn{2}{|c|}{ Total } & \multirow[b]{2}{*}{ Results } \\
\hline & No & $\%$ & No & $\%$ & No & $\%$ & No & $\%$ & \\
\hline Anomic & 2 & 10.0 & 0 & 0 & 0 & 0.0 & 2 & 10.0 & $x^{2}=16.306$ \\
\hline Broca's & 0 & 0.0 & 1 & 5.0 & 2 & 10.0 & 3 & 15.0 & $\mathrm{df}=10$ \\
\hline Global & 4 & 20.0 & 1 & 5.0 & 5 & 25.0 & 10 & 50.0 & $P=0.091$ \\
\hline$T / C$ sensory & 3 & 15.0 & 0 & 0.0 & 0 & 0.0 & 3 & 15.0 & \\
\hline TCM & 0 & 0.0 & 1 & 5.0 & 0 & 0.0 & 1 & 5.0 & \\
\hline \multirow[t]{2}{*}{ Wernicke's } & 0 & 0.0 & 0 & 0.0 & 1 & 5.0 & 1 & 5.0 & \\
\hline & 9 & 45.0 & 3 & 15.0 & 8 & 40.0 & 20 & 100.0 & \\
\hline
\end{tabular}


From Table 6, we observe that the frequencies of Anomic, Broca, Global, T/C sensory, T/C Motor and Wernickes aphasia were $10 \%, 15 \%, 50 \%, 15 \%, 5 \%$ and $5 \%$, respectively.

Table-7, states the frequency of the different classes of aphasia in our study. Out of the aphasics, $30 \%$ were fluent, and $70 \%$ were nonfluent

Table-8, categorizes the AQ values from our study. Among them, the values were normal in $60 \%$ and mild aphasia was $4 \%$, moderate aphasia was $12 \%$ and severe aphasia was $24 \%$ (Fig. 3)
From Table 9 Figure 4 we can infer the association of left side lesions with aphasia incidence $(p<0.05)$

\section{Results}

In our study (Fig. 1),

- The mean age of females was observed to be $58.1 \pm 11.3$ years.

- The mean age of males was $70.0 \pm 7.7$ years.

- The range of ages was 38-79 years.

Table 6: Types of aphasia

\begin{tabular}{lccc}
\hline S. No. & Types of aphasia & Frequency & Percentage \\
\hline 1 & Anomic & 2 & 10.0 \\
2 & Broca's & 3 & 15.0 \\
3 & Global & 10 & 50.0 \\
4 & T/C sensory & 3 & 15.0 \\
5 & TCM & 1 & 5.0 \\
6 & Wernicke's & 1 & 5.0 \\
Total & & 20 & 100.0 \\
\hline
\end{tabular}

Table 7: Class of aphasia

\begin{tabular}{llll}
\hline S. No & \multicolumn{1}{c}{ Types of aphasia } & Frequency & Percentage \\
\hline 1 & Fluent & 6 & 30.0 \\
2 & Nonfluent & 14 & 70.0 \\
Total & & 20 & 100.0 \\
\hline
\end{tabular}

Table 8: Category of aphasia according to the $A Q$ values

\begin{tabular}{lcccc}
\hline S. No. & Severity & AQ values & Frequency & \% \\
\hline 1 & Normal & 93.8 & 30 & 60.0 \\
2 & Mild & $75-93.8$ & 2 & 4.0 \\
3 & Moderate & $50-74$ & 6 & 12.0 \\
4 & Severe & $<50$ & 12 & 24.0 \\
Total & & & 50 & 100.0 \\
\hline
\end{tabular}

Table 9: Association of aphasia incidence with lesion side

\begin{tabular}{|c|c|c|c|c|c|c|c|}
\hline \multirow[b]{2}{*}{ Insult side } & \multicolumn{2}{|c|}{ Present } & \multicolumn{2}{|c|}{ Absent } & \multicolumn{2}{|c|}{ Total } & \multirow[b]{2}{*}{ Significance } \\
\hline & No & $\%$ & No & $\%$ & No & $\%$ & \\
\hline$B / L$ & 0 & 0.0 & 1 & 3.3 & 1 & 2.0 & $x^{2}=12.508$ \\
\hline Left & 19 & 95.0 & 14 & 46.7 & 33 & 66.0 & $\begin{array}{c}\mathrm{df}=2 \\
P=0.002\end{array}$ \\
\hline Right & 1 & 5.0 & 15 & 50.0 & 16 & 32.0 & \\
\hline Total & 20 & 100.0 & 30 & 100.0 & 50 & 100.0 & \\
\hline
\end{tabular}




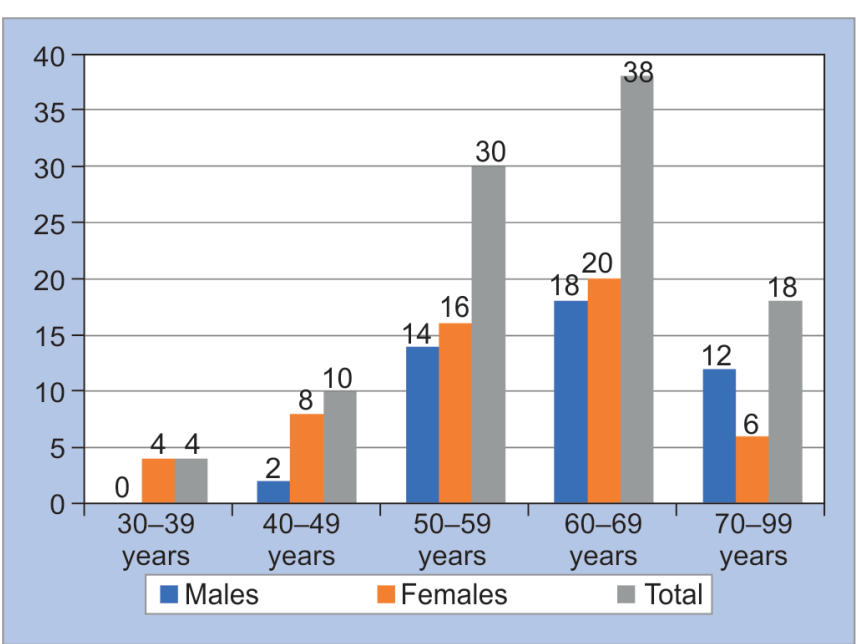

Fig. 1: Representation of ages of subjects

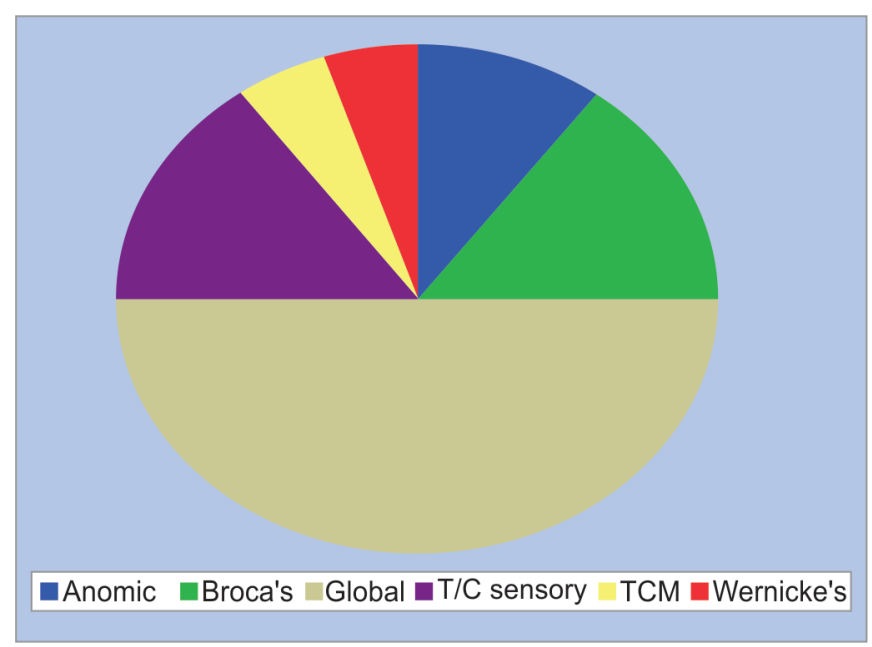

Fig. 2: Distribution of types of aphasia

\section{Discussion}

- In our study, the ages of patients lie between 38 and 80 years of age, with a maximum number in the age group of 51-60 years (36.7\%). All patients were right-handed.

In our study, there was a greater number of patients with Infarction as the pathologic process, than the ones with Hemorrhage as the pathologic process. In a similar study done for poststroke aphasia, the ischemic stroke had predominance. ${ }^{36}$

Pure subcortical lesions were seen in $40 \%$ of our cases. In our study young stroke (stroke in patient under 50 years of age), was present in seven cases (14\%) which was lower than the count seen in another earlier study. ${ }^{36}$

- Aphasia was present in 20 patients (40\%). In similar studies, the frequency of poststroke Aphasia ranged between 21 and 38\%. ${ }^{37}$ In our study, there was no significant association between the type of vascular insult and the type of aphasia. A similar result was seen in another study in patients with stroke-related aphasia. ${ }^{36}$

Our study had one patient with crossed aphasia (4\%). In earlier stroke studies, crossed aphasia had an observed prevalence between 0.4 and $3.5 \%$. $^{38,39}$

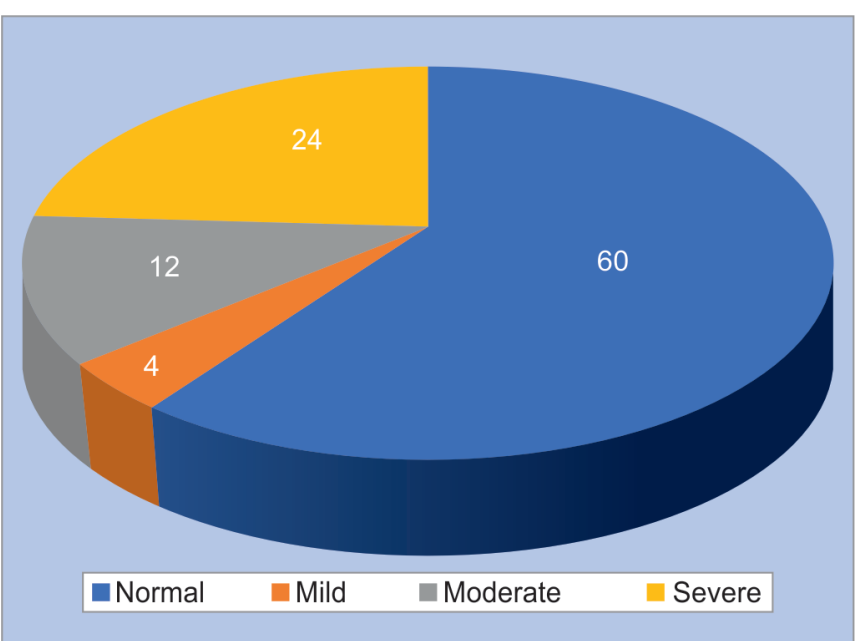

Fig. 3: Categories of aphasia

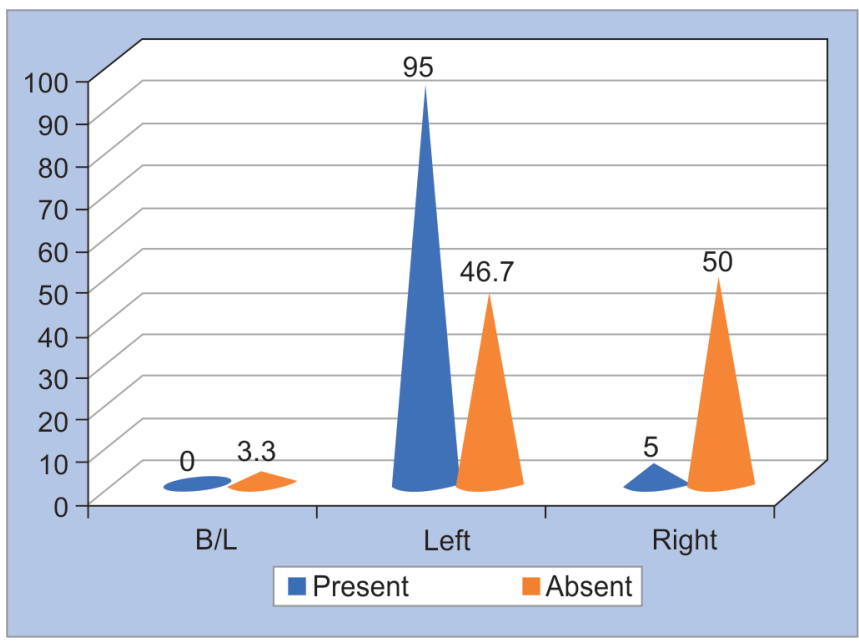

Fig. 4: Association of aphasia incidence with lesion side

- In our study, nonfluent aphasia was predominant, with the descending order of frequencies of the subtypes being:

- Global aphasia (50\%)

- Broca's aphaisa (15\%)

- Transcortical sensory aphasia (15\%),

- Anomic aphasia(10\%),

- Transcortical motor aphasia(5\%),

- Wernicke's aphasia(5\%)

- Conduction aphasia- Nil.

Overall, $70 \%$ of the aphasics in our study had nonfluent aphasia. Prior studies ${ }^{39-41}$ have also reported a similar incidence of nonfluent aphasia.

Anomicaphasia had afrequency of $16-25 \%$ in earlier studies ${ }^{40,42}$ but was seen in only $10 \%$ of cases in our study. The frequency of other subtypes was also seen to differ from earlier studies.

- Using the Aphasia Quotient derived from WAB to classify the aphasia in the affected patients, we had a greater number of cases in the "Severe " grade. An earlier study had similar results. ${ }^{36}$

- In our study, the site of stroke and the observed type of aphasia were not correlated to any significant extent. Certain earlier 
studies in stroke patients ${ }^{42-44}$ had seen a correlation between the aphasia type and location of lesion, while certain other studies did not. ${ }^{45,46}$

\section{ConcLusion}

- The frequency of poststroke aphasia was higher in our study (40\%) than that seen in earlier studies.

- Based on the Aphasia Quotient obtained from the Western Aphasia Battery, we categorized the aphasia as mild (4\%), moderate (12\%), and severe (24\%).

- The aphasia types observed in our study were:

- Anomic (10\%)

- Broca (15\%)

- Global (50\%)

- Transcortical sensory (15\%)

- Transcortical motor (5\%)

- Wernicke's aphasia (5\%).

- Vascular insult type and the variety of aphasia present were not associated significantly.

- We have seen a preponderance of nonfluent aphasia in our study with global aphasia (50\%) being the commonest subtype.

- We had also attempted to determine the presence of any association between the localization of lesion and type of aphasia. However, no such significant association was found.

- A significant association was detected between lesions located topographically on the left side and the occurrence of aphasia.

\section{Limitations}

- The study was retrospective in nature.

- In our study, we divided the lesions according to clinical features and therefore lesion classification depended on the investigator's discretion.

- The study would have been more useful if the functional recovery and other characteristics associated with the subitems were investigated with a follow-up visit with patients who showed various aphasias.

- In our study, we had not given weightage to lesion volume and severity.

- Our study had only right-handed patients.

\section{References}

1. National Stroke Association Stroke 101 fact sheet. 2011.

2. Roger VL, Go AS, Lloyd-Jones DM, et al. Heart disease and stroke statistics-2011 update: a report from the American Heart Association. Circulation 2011;123(4):e18-e209. DOI: 10.1161/CIR.0b013e3182009701

3. Dombovy M. Stroke: clinical course and neurophysiologic mechanisms of recovery. Crit Rev Phys Rehabil Med 1998;12(17):171-188.

4. Annett M. Hand preference and the laterality of cerebral speech. Cortex 1975;11(4):305-328. DOI: 10.1016/s0010-9452(75)80024-4

5. Parikh RM, Lipsey, JR, Robinson RG, et al. Two-year longitudinal study of post-stroke mood disorders: dynamic changes in correlates of depression at one and two years. Stroke 1987;18(3):579-584. DOI: 10.1161/01.str.18.3.579

6. Pedersen PM, Vinter K, Olsen TS. Aphasia after stroke: type, severity and prognosis. The Copenhagen aphasia study. Cerebrovasc Dis 2004;17(1):35-43. DOI: 10.1159/000073896

7. Choi-Kwon S, Kim HS, Kwon SU, et al. Factors affecting the burden on caregivers of stroke survivors in South Korea. Arch Phys Med Rehabil 2005;86(5):1043-1048. DOI: 10.1016/j.apmr.2004.09.013
8. O'Brien, M. P., PJ. (1978). Total Care of the Stroke Patient: Little Brown and Co.

9. Dickey L, Kagan A, Lindsay MP, et al. Incidence and profile of inpatient stroke-induced aphasia in Ontario, Canada. Arch Phys Med Rehabil 2010;91(2):196-202. DOI: 10.1016/j.apmr.2009.09.020

10. Inatomi Y, Yonehara T, Omiya $S$, et al. Aphasia during the acute phase in ischemic stroke. Cerebrovasc Dis 2008;25(4):316-323. DOI: 10.1159/000118376

11. Ryglewicz D, Hier D B, Wiszniewska M, et al. Ischemic strokes are more severe in Poland than in the United States. Neurology 2000;54(2): 513-515. DOI: 10.1212/wnl.54.2.513

12. Kadojić $D$, Bijelić $B R$, Radanović $R$, et al. Aphasia in patients with ische- mic stroke. ActaClin Croat 2012;51(2):221-225.

13. Feigin V L, Krishnamurthi R V, Parmar $P$, et al. Update on the global burden of ischemic and hemorrhagic stroke in 1990-2013: The GBD 2013 study. Neuroepidemiology 2015;45(3):161-176. DOI: $10.1159 / 000441085$

14. Watila $M$ M, Balarabe $S$ A. Factors predicting post-stroke aphasia recovery. J Neurol Sci 2015:352(1-2):12-18. DOI: 10.1016/j. jns.2015.03.020

15. Heiss WD, Thiel A, Kessler J, et al. Disturbance and recovery of language function: correlates in PET activation studies. Neuroimage 2003;20((Suppl 1)):S42-S49. DOI: 10.1016/j.neuroimage. 2003.09.005

16. Saur D, Lange R, Baumgaertner $A$, et al. Dynamics of lan- guage reorganization after stroke. Brain 2006;129(Pt 6):1371-1384. DOI: 10.1093/brain/awl090

17. LaPointe L. Handbook of aphasia and brain-based cognitivelanguage disorders. New York: Thieme Medical Publishers; 2011

18. Hantson L, De Weerdt W, De Keyser J, et al. The European Stroke Scale. Stroke 1994;25(11):2215-2219. DOI: 10.1161/01.str.25.11.2215

19. Cote R, Hachinski VC, Shurvell BL, et al. The Canadian Neurological Scale: a prelim- inary study in acute stroke. Stroke 1986;17(4):731-737. DOI: 10.1161/01.str.17.4.731

20. Goldstein LB, Bertels C, Davis JN. Interrater reliability of the NIH stroke scale. Arch Neurol 1989;46(6):660-662. DOI: 10.1001/ archneur.1989.00520420080026

21. El Hachioui $H$, Visch-Brink E G, de LauL $M$, et al. Screening tests for aphasia in patients with stroke: a systematic review. J Neurol 2017;264(2):211-220. DOI: 10.1007/s00415-016-8170-8

22. Enderby P M, Wood V A, Wade D T, et al. The Frenchay Aphasia Screening Test: a short, simple test for aphasia appropriate for non-specialists. Int Rehabil Med 1987;8(4):166-170. DOI: 10.3109/03790798709166209

23. Flamand-Roze C, Falissard B, Roze E, et al. Validation of a new language screening tool for patients with acute stroke: the Language Screening Test (LAST). Stroke 2011;42(5):1224-1229. DOI: 10.1161/ STROKEAHA.110.609503

24. Al-Khawaja I, Wade DT, Collin CF. Bedside screening for aphasia: a comparison of two methods. J Neurol 1996;243(2):201-204. PMID: 8750561 DOI: 10.1007/BF02444015

25. Thommessen B, Thoresen GE, Bautz-Holter E, et al. Screening by nurses for aphasia in stroke: The Ullevaal Aphasia Screening (UAS) Test. Disabil Rehabil 1999;21(3):110-115. DOI: 10.1080/096382899297846

26. Flowers HL, Skoretz SA, Silver FL, et al. Poststroke aphasia frequency, recovery, and outcomes: a systematic review and meta-analysis. Arch Phys Med Rehabil 2016;97(12): 2188-2201.e8. DOI: 10.1016/j. apmr.2016.03.006

27. Crary MA, Haak NJ, Malinsky AE. Preliminary psychometric evaluation of an acute aphasia screening protocol. Aphasiology 1989;3(7): 611-618. DOI: 10.1080/02687038908249027

28. Kertesz A. Western Aphasia Battery-Revised. San Antonio, TX: Pearson; 2007.

29. Christine J, Clark W, Mortensen L. Mount Wilga High Level Language Test (Revised): Administration \& scoring manual plus test form with UK adaptions and large print additions. Hornsby, NSW: Mount Wilga Rehabilitation Centre; 1986. 
30. Sklar M. Sklar Aphasia Test. Los Angeles, CA: Western Psychological Services; 1973.

31. Willmes K, Poeck K, Weniger D, . Facet theory applied to the construction and validation of the Aachen Aphasia Test et al. Brain Lang 1983;18(2):259-276. DOI: 10.1016/0093-934x(83)90020-2

32. West JF, Sands ES, Ross-Swain D. Bedside Evaluation Screening Test: 2nd Edition (BEST-2). Austin, TX: Pro-Ed; 1998.

33. Keenan JS, Brassell EG. Aphasia Language Performance Scales (Spanish Version). Murfreesboro, TN: Pinnacle Press; 1975.

34. Whurr R. Aphasia screening test a multi-dimensional assessment procedure for adults with acquired aphasia, Milton Keynes, UK: Speechmark Publishing; 2011.

35. Shewan CM, Kertesz A. Reliability and validity characteristics of the Western Aphasia Battery (WAB). J Speech Hear Disord 1980;45(3): 308-324. DOI: 10.1044/jshd.4503.308

36. Bohra V, Khwaja GA, Jain S, et al. Clinicoanatomical correlation in stroke related aphasia. Ann Indian Acad Neurol 2015;18(4):424-429. DOI: 10.4103/0972-2327.165469

37. Nadamuni S. Researchers identify stroke subtypes in India. Lancet 2002;359((9305)):500. DOI: 10.1016/s0140-6736(02)07702-4

38. Alexander MP, Annett M. Crossed aphasia and related anomalies of cerebral organization: case reports and a genetic hypothesis. Brain Lang 1996;55(2):213-239. DOI: 10.1006/brln.1996.0102
39. Carr MS, Jacobson T, Boller F. Crossed aphasia: analysis of four cases. Brain Lang 1981;14(1):190-202. DOI:10.1016/0093-934x(81)90075-4

40. Godefroy O, Dubois C, Debachy B, et al. Vascular aphasias: main characteristics of patients hospitalized in acute stroke units. Stroke 2002;33(3):702-705. DOI: 10.1161/hs0302.103653

41. Croquelois A, Bogousslavsky J. Stroke aphasia: 1,500 consecutive cases. Cerebrovasc Dis 2011;31(4):392-399. DOI: 10.1159/000323217

42. Kreisler A, Godefroy O, Delmaire C, et al. The anatomy of aphasia revisited. Neurology 2000;54(5):1117-1123. DOI: 10.1212/ wnl.54.5.1117

43. Yang $\mathrm{ZH}$, Zhao $\mathrm{XQ}$, Wang $\mathrm{CX}$, et al. Neuroanatomic correlation of the post-stroke aphasias studied with imaging. Neurol Res 2008;30(4):356-360. DOI: 10.1179/174313208X300332

44. Hayward RW, Naeser MA, Zatz LM. Cranial computed tomography in aphasia. Correlation of anatomical lesions with functional deficits. Radiology 1977;123(3):653-660. DOI: 10.1148/123.3.653

45. Josephs KA, Duffy JR, Strand EA, et al. Clinicopathological and imaging correlates of progressive aphasia and apraxia of speech. Brain 2006;129(Pt 6):1385-1398. DOI: 10.1093/brain/awl078

46. Colombo A, Sorgato P, Scarpa M. Language disturbances following vascular lesions restricted to the left basal ganglia, thalamus, and white matter. Neuropsychology 1989;3(2):75-80. DOI: 10.1037/ h0091762 